\title{
Power-Law Behavior of Power Spectra in Low Prandtl Number Rayleigh-Bénard Convection
}

\author{
M. R. Paul* and M.C. Cross \\ Department of Physics, California Institute of Technology 114-36, Pasadena, California 91125 \\ P. F. Fischer \\ Mathematics and Computer Science Division, Argonne National Laboratory, Argonne, Illinois 60439 \\ H. S. Greenside \\ Department of Physics, Duke University, Durham, North Carolina 27706
}

(Received 2 May 2001; published 25 September 2001)

\begin{abstract}
The origin of the power-law decay measured in the power spectra of low Prandtl number RayleighBénard convection near the onset of chaos is addressed using long time numerical simulations of the three-dimensional Boussinesq equations in cylindrical domains. The power law is found to arise from quasidiscontinuous changes in the slope of the time series of the heat transport associated with the nucleation of dislocation pairs and roll pinch-off events. For larger frequencies, the power spectra decay exponentially as expected for time continuous deterministic dynamics.
\end{abstract}

DOI: 10.1103/PhysRevLett.87.154501

Significant insight into the onset of chaotic dynamics in fluid systems, and continuum systems in general, has been gained from cryogenic Rayleigh-Bénard convection experiments [1-4]; for a review, see [5,6]. Two of the most dramatic discoveries were the observation of time dependence almost immediately above the onset of convective flow, and the power-law falloff in frequency for the power spectral density derived from time series of a global measurement of the temperature difference across the fluid at fixed heat flow [1]. However, these and other important observations remain poorly understood although further insight has been gained from room temperature argon experiments allowing flow visualization [7-9]. The power-law behavior is unexpected, since bounded deterministic models typically show an exponential falloff at high frequency [10]. Phenomenological stochastic models were proposed to explain the spectra [11,12], but no understanding of the origin of the ad hoc stochastic driving has followed.

In this Letter, we use numerical simulations of the threedimensional Boussinesq equations for the fluid flow and heat transport in the cylindrical geometries of the experiments with realistic boundary conditions to investigate the power spectrum in more detail. The numerical simulations allow us to determine the spatial structure of the flow field in the aperiodic dynamics, and the absence of experimental or measurement noise provides us with more complete results for the power spectra. Our completely deterministic simulations yield results consistent with the experimental observations, including a power-law falloff of the power spectrum over the range accessible to the experiment. Using knowledge of the flow field, we are able to associate this power-law behavior with specific events in the dynamics, namely, the creation and annihilation of defects in the convection roll structure, which occur on a time scale rapid compared with the slow pattern evolution. At higher frequencies, the power spectra decay exponentially, consis-
PACS numbers: 47.54. $+\mathrm{r}, 47.20 . \mathrm{Bp}, 47.27 . \mathrm{Te}, 47.52 .+\mathrm{j}$

tent with the behavior expected for smooth deterministic time evolution. The low amplitude region of the spectra was inaccessible experimentally due to the noise floor.

Our simulations in a cylindrical geometry are performed using an efficient spectral element algorithm (described in detail elsewhere [13]). The velocity $\vec{u}$, temperature $T$, and pressure $p$ evolve according to the Boussinesq equations,

$$
\begin{aligned}
\sigma^{-1}\left(\partial_{t}+\vec{u} \cdot \vec{\nabla}\right) \vec{u} & =-\vec{\nabla} p+R T \hat{z}+\nabla^{2} \vec{u}, \\
\left(\partial_{t}+\vec{u} \cdot \vec{\nabla}\right) T & =\nabla^{2} T, \\
\vec{\nabla} \cdot \vec{u} & =0,
\end{aligned}
$$

where $\partial_{t}$ indicates time differentiation, $\hat{z}$ is a unit vector in the vertical direction, $\sigma$ is the Prandtl number, and $R$ is the Rayleigh number. The equations are nondimensionalized in the standard manner using the layer depth $h$, the vertical diffusion time for heat $\tau_{v}$, and the constant temperature difference across the layer $\Delta T$, as the length, time, and temperature scales, respectively. All variables in the following discussion are nondimensional using this scaling. The lower and upper surfaces $(z=0,1)$ are no slip and are held at constant temperature. The sidewalls are no slip and perfectly conducting [14], and the initial conditions are small random thermal perturbations of magnitude $0.2 \mathrm{im}$ posed upon an otherwise quiescent layer, $\vec{u}=0, T=0$.

In nearly all cryogenic experiments, for reasons of increased experimental resolution, the heat flux across the convection layer, $Q$, and the temperature of either the upper or lower surface are held constant while measurements of $\Delta T(t)$ are made. These measurements are reported as $R(t) / R_{c}$ or $\Delta T(t) / \Delta T_{c}$, where $\Delta T_{c}$ is the temperature difference across the layer and $R_{c}$ is the Rayleigh number at the convective threshold. Theoretical calculations, on the other hand, most often consider both the upper and lower surfaces to be held at constant temperature and observe the 
time dependence in $Q(t)$, which can be reported as a time series of the Nusselt number $N(t)$ (the normalized heat current through the fluid layer). It has been shown experimentally that fixing $Q$ or fixing $\Delta T$ does not appear to change the flow dynamics, and the conclusions from measurements of $R(t)$ at fixed $N$ or $N(t)$ at fixed $R$ will be similar [16].

In order to make contact with experiment $[3,16,17]$ we focus our discussion on simulations with aspect ratio $\Gamma=$ $4.72(\Gamma=r / h, r$ is the radius), $\sigma=0.78$ (experimental fluid was nonsuperfluid $\mathrm{He}^{4}$ ), and constant $\Delta T$. A key result of the experiments was the observation that the power spectrum, $P(\nu)$, of measured $R(t)$ values exhibited the power-law behavior, $P(\nu) \sim \nu^{-n}, n=-4.0 \pm 0.2$ over the frequency range $0.5 \lesssim \nu \lesssim 9$ [17] (results were reported for $\epsilon=3.62$, where $\epsilon=\left(R-R_{c}\right) / R_{c}$ is the reduced Rayleigh number).

Six representative time series $N(t)$ from our simulations are shown in Fig. 1. In terms of the horizontal diffusion time for heat $\tau_{h}\left(\tau_{h}=\Gamma^{2} \tau_{v}\right)$, the simulation times are $t_{f} \approx 100 \tau_{h}\left[t_{f} \approx 50 \tau_{h}\right.$ for case (vi)], which is comparable with the longest experiments, $t_{f} \approx 65 \tau_{h}$ (with one long run for $t_{f} \approx 135 \tau_{h}$ ) [3]. This is considerably longer than $\Gamma \tau_{h}$, which has been suggested as the earliest time scale for the flow field to reach equilibrium [18]. However, as discussed below, we find that the dynamics can occur on even longer time scales. The simulated time-averaged values of $N-1$ are within $5.5 \%$ of the experimental values given by $N-1=1.034 \beta+$ $0.981 \beta^{3}-0.866 \beta^{5}, \beta=1-R_{c} / R$ [1]. Spatial and temporal resolution studies have been performed to ensure the accuracy of the calculated values of $N(t)$ for the chosen simulation parameters.

We now consider the periodic time series in more detail, case (ii) in Fig. 1. To determine the influence of the pattern

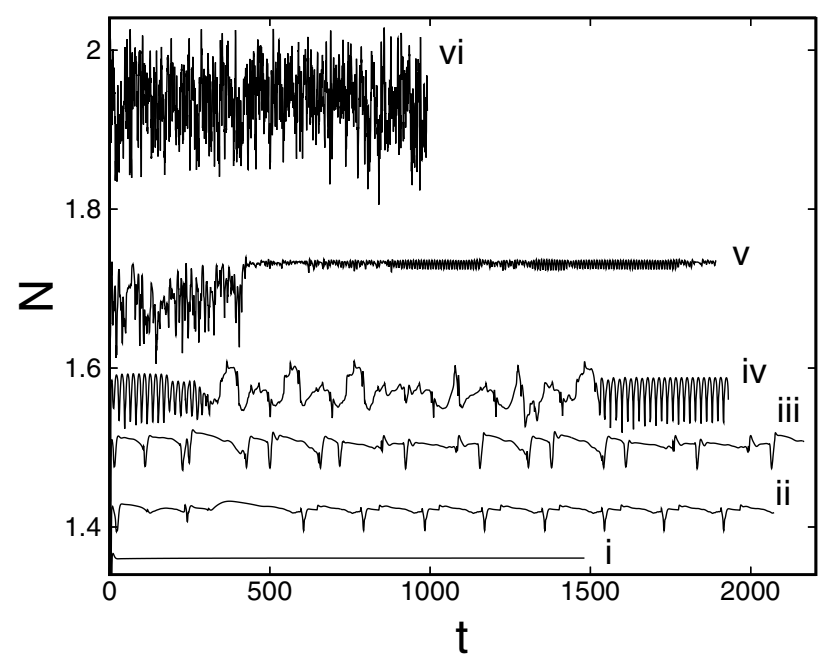

FIG. 1. Plots of the dimensionless heat transport $N(t)$ for cases (i-vi) for reduced Rayleigh number $\epsilon=0.557,0.614,0.8,1.0$, 1.5 , and 3.0, respectively. For cases (i-v), $\Delta t=0.01$, and for case (vi), $\Delta t=0.005$ ( $\Delta t$ is the time step). dynamics on the power spectrum, we used a sliding window in time to calculate successive time-localized power spectra (a spectrogram) as shown in the lower panel of Fig. 2. Most of the power in the spectra can be attributed to nucleation of dislocation pairs (and, to a lesser extent, dislocation annihilation). A plot of $P(\nu)$ at a particular time (a vertical slice of the lower panel of Fig. 2) yields the windowed power spectrum centered about that point in time in the upper panel of Fig. 2. Figure 3 shows three such power spectra from the spectrogram evaluated with windows centered on (a), (d), and (e) corresponding to dislocation nucleation, annihilation, and glide, respectively. The local power spectrum centered on the nucleation of a dislocation pair generates a power-law region of significant magnitude; the local power spectrum centered on the dislocation annihilation generates a power-law region that is a factor of 10 smaller in magnitude, whereas the local power spectrum calculated during dislocation glide falls off more rapidly with $\nu$ and does not make a significant contribution. The origin of the power law is the time signature of the nucleation of a dislocation pair manifested in the quasidiscontinuous slope of $N$ during the rapid excursion to a state of decreased heat transfer (an individual event is shown in Fig. 4). Note that a triangular feature with discontinuous changes in the slope yields a $\nu^{-4}$ asymptotic behavior in the power spectrum.

The power spectra of the remaining time series in Fig. 1 yield a power-law region $P(\nu) \sim \nu^{-4}$ because the dynamics (periodic, quasiperiodic, and chaotic) are dominated by the nucleation of dislocation pairs and roll pinch-off events. The differences between the chaotic and periodic

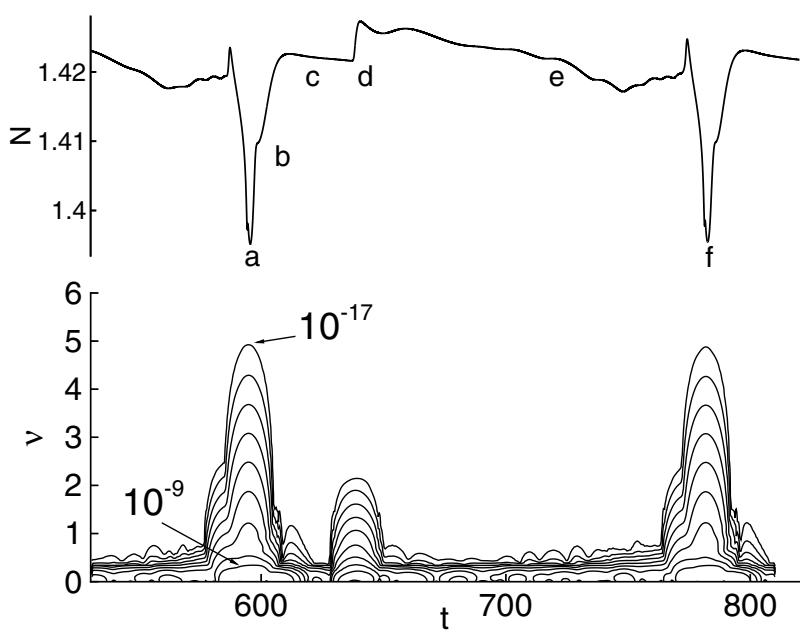

FIG. 2. Time series $N(t)$ (top) and corresponding spectrogram (bottom) for one period of case (ii). The labels $a-f$ represent particular moments in the evolution of the pattern and are discussed in the text. The spectrogram displays 9 orders of magnitude of the power, $P(\nu)$, with the smallest and largest contours labeled; the remaining contours each differ by a factor of 10 . The spectrogram was calculated using a sliding Hann window of width $\Delta t=20.48$ and linearly detrended overlapping segments (segments overlap by $t=20.0$ ). 


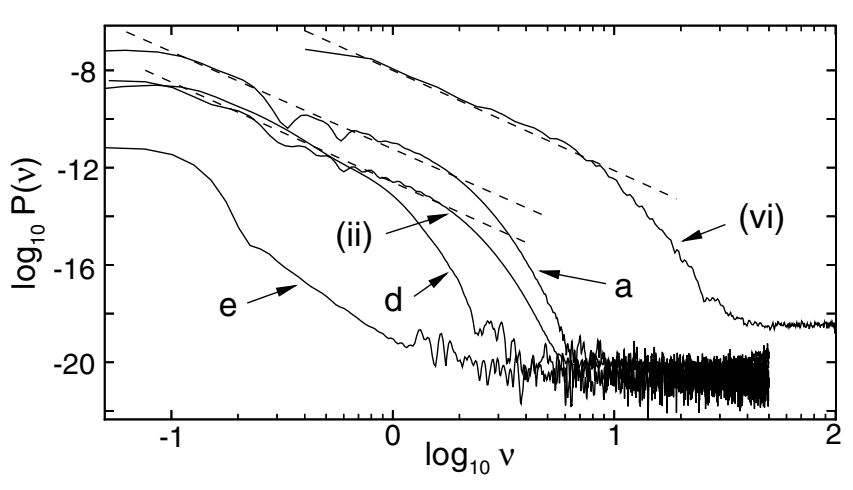

FIG. 3. Windowed power spectra. The power spectra labeled (a), (d), and (e) are vertical slices of the spectrogram taken at representative times for case (ii) at dislocation nucleation, dislocation annihilation, and dislocation glide, respectively. The curves labeled (ii) and (vi) are the average of the windowed power spectra using the entire spectrogram for cases (ii) and (vi). The dashed lines represent $P(\nu) \sim \nu^{-4}$.

dynamics are apparent in the low-frequency region and are more apparent on a log-linear plot of $P(\nu)$. For example, Fig. 4 shows the similarity between $N(t)$ for the periodic simulation, case (ii), and the chaotic simulation, case (vi). The dynamics in the chaotic state are much more complicated; however, they are dominated by the roll pinch-off events that maintain the characteristic quasidiscontinuous slope of $N$, yielding a power-law region in the power spectrum. A comparison of the power spectra for the periodic and chaotic time series is shown in Fig. 3.

The average of the windowed power spectra of Fig. 3 eventually exhibit an exponential decay, which continues

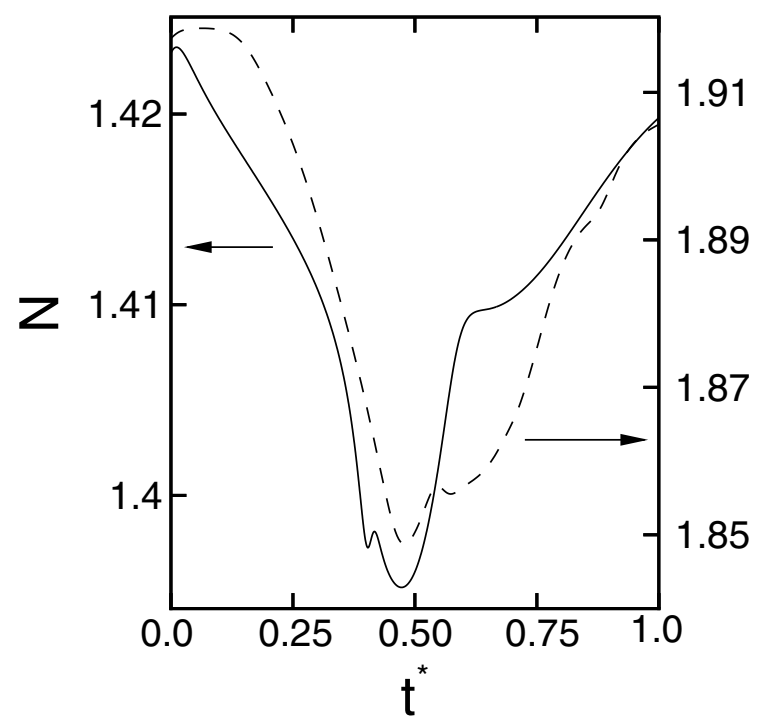

FIG. 4. A closeup of the time series $N(t)$, illustrating the signature of a nucleation of a dislocation pair for case (ii) (solid line), and the signature of a roll pinch-off for case (vi) (dashed line). Time $t^{*}$ is measured as $t^{*}=\left(t-t_{i}\right) / \Delta t ; t_{i}$ denotes when the event begins, and $\Delta t$ is the duration of the event. For the events shown, $t_{i}=587,270.5$ and $\Delta t=18,1.5$ for cases (ii) and (vi), respectively. until reaching the spectrum floor; this is shown for case (ii) in Fig. 5. Exponential decay in the power spectra at high frequency is expected for bounded smooth deterministic dynamics [10]. The exponential decay in the power spectra was not detected in experiment due to the presence of instrumental noise which masked the small scale region.

In the cryogenic experiments, flow visualization was not possible leaving the precise details of the underlying pattern uncertain. With this in mind, we briefly discuss the dynamics represented in Fig. 1. Case (i) illustrates a timeindependent Pan-Am pattern similar to panel (a) of Fig. 6. Case (ii) is periodic with period $t=8.4 \tau_{h}$ (note the initial transient lasting $27 \tau_{h}$ ); the dynamics of one period are illustrated in Figs. 2 and 6. Figure 6 displays the pattern at six different instances in time corresponding to the events labeled in the upper panel of Fig. 2. Initially there is a Pan-Am pattern with two opposing wall foci causing roll compression $(a)$, eventually nucleating a dislocation pair in the center of the domain $(b)$. The dislocations quickly climb to the wall $(c)$, at which point they both begin to glide slowly toward the same wall focus. However, the lower dislocation is annihilated at the sidewall $(d)$, and the remaining dislocation continues to glide slowly into the wall focus, where it is annihilated $(e)$. A Pan-Am pattern again forms $(f)$, and finally the process repeats. This is in general agreement with flow visualizations from related room-temperature argon-based experiments [7-9]. Case (iii) may be periodic on a long time scale of $t \approx 40 \tau_{h}$; the duration of the simulation is inadequate to be conclusive. Case (iv) illustrates a chaotic burst of duration $t \approx 54 \tau_{h}$ bounded by periodic dynamics with a period of $t \approx 17$. Again the simulation duration is inadequate to determine whether this is a transient state or whether the chaotic bursting will repeat. Case (v) shows an initial chaotic transient that makes a transition at $t \approx 18 \tau_{h}$ to a very complicated quasiperiodic state where the central roll pair is pinned by the dynamic motion of two opposing disclinations. The dominant mode in the quasiperiodic state has a time scale $t \approx 8$. Case (vi) illustrates chaotic dynamics.

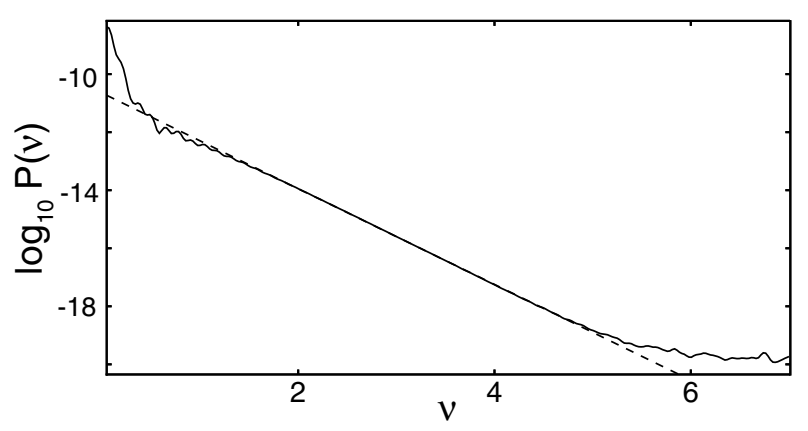

FIG. 5. The power spectrum, $\langle P(\nu)\rangle$, for case (ii) on a loglinear scale to illustrate the region of exponential decay. The slope of the dashed line is -3.8 ; the crossover to exponential decay occurs at $\nu \approx 1.5$. 

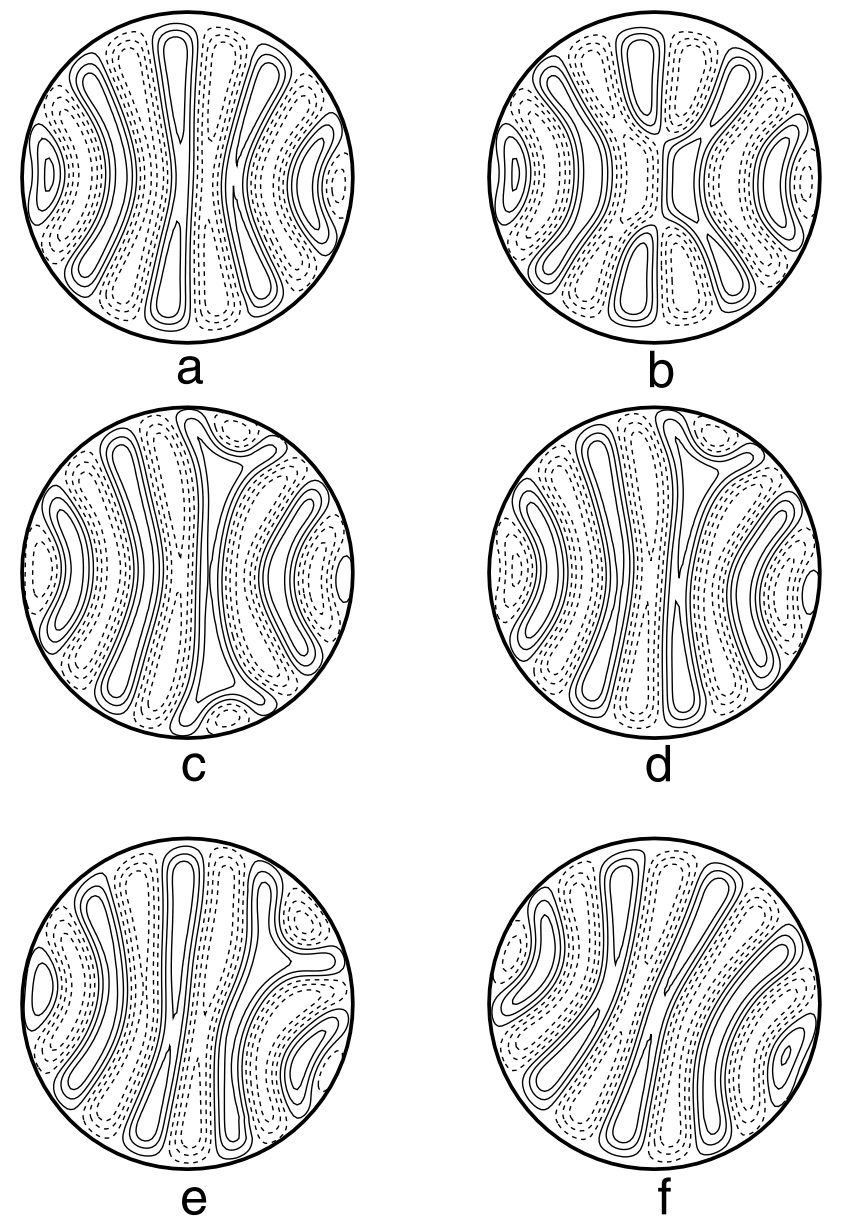

FIG. 6. Flow visualization showing contours of the thermal perturbation at the mid-depth, (six evenly spaced contours; $-0.2 \leq \delta T \leq 0.2$, negative values are dashed lines, and positive values are solid lines) for case (ii). Panels (a)-(f) are for $t=600,605,630,650,735,785$. The dislocations glide to the right; during the next period, the dislocations glide to the left, as can already be discerned in (f) by the bias in the roll compression. This left and right alternation continues for the entire simulation.

We have also performed simulations for the $\Gamma=4.72$ cylindrical domain with insulating lateral boundary conditions, in addition to simulations in a $\Gamma=7.66$ domain ( $\sigma=0.69$ for argon) for both conducting and insulating lateral boundaries. Considering these additional results, we maintain our conclusions concerning the origin of the power law.

This work represents a joint computational and theoretical effort to further our quantitative understanding of complex dynamics in spatially extended nonequlibrium systems. An important link missing in nearly all theoreti- cal work to date has been a quantitative comparison with experiment. Our results demonstrate that this quantitative comparison with experiment is now possible. We plan to use this approach to investigate spatiotemporal chaos in larger aspect ratio systems.

This research was supported by the U.S. Department of Energy, Grant No. DE-FT02-98ER14892, and the Mathematical, Information, and Computational Sciences Division subprogram of the Office of Advanced Scientific Computing Research, U.S. Department of Energy, under Contract No. W-31-109-Eng-38. We also acknowledge the Caltech Center for Advanced Computing Research and the North Carolina Supercomputing Center.

*Electronic address: mpaul@caltech.edu

[1] G. Ahlers, Phys. Rev. Lett. 33, 1185 (1974).

[2] G. Ahlers and R. P. Behringer, Phys. Rev. Lett. 40, 712 (1978).

[3] G. Ahlers and R.W. Walden, Phys. Rev. Lett. 44, 445 (1980).

[4] A. Libchaber and J. Maurer, J. Phys. (Paris), Lett. 39, 369 (1978).

[5] M. C. Cross and P. C. Hohenberg, Rev. Mod. Phys. 65, 851 (1993).

[6] R. P. Behringer, Rev. Mod. Phys. 57, 657 (1985).

[7] A. Pocheau, V. Croquette, and P. Le Gal, Phys. Rev. Lett. 55, 1094 (1985).

[8] V. Croquette, P. Le Gal, and A. Pocheau, Phys. Scr. T13, 135 (1986).

[9] A. Pocheau, J. Phys. (Paris) 50, 2059 (1989).

[10] U. Frisch and R. Morf, Phys. Rev. A 23, 2673 (1981).

[11] H. S. Greenside, G. Ahlers, P. C. Hohenberg, and R. W. Walden, Physica (Amsterdam) 5D, 322 (1982).

[12] S. Zaleski, Phys. Rev. A 39, 3088 (1989).

[13] P. F. Fischer, J. Comput. Phys. 133, 84 (1997).

[14] The cryogenic experiments [15] were bounded by a thin stainless steel lateral wall separating the convective layer from a surrounding vacuum resulting in the diversion of $\sim 20 \%$ of the heat flow suggesting an insulating boundary. The room-temperature argon experiments [8,9], on the other hand, were bounded by highly conducting sidewalls. We have performed simulations using both lateral boundary conditions and find that the general nature of the resulting dynamics is unaffected.

[15] R. P. Behringer and G. Ahlers, J. Fluid Mech. 125, 219 (1982).

[16] H. Gao and R. P. Behringer, Phys. Rev. A 30, 2837 (1984).

[17] G. Ahlers and R. P. Behringer, Prog. Theor. Phys. Suppl. 64, 186 (1978).

[18] M. C. Cross and A. C. Newell, Physica (Amsterdam) 10D, 299 (1984). 